\title{
Does Stress Reduction Change the Levels of Cortisol Secretion in Patients With Coronary Artery Disease?
}

Mireille Rydén, Bo Hedbäck and Lena Jonasson

\section{Linköping University Post Print}

N.B.: When citing this work, cite the original article.

Original Publication:

Mireille Rydén, Bo Hedbäck and Lena Jonasson, Does Stress Reduction Change the Levels of Cortisol Secretion in Patients With Coronary Artery Disease?, 2009, JOURNAL OF CARDIOPULMONARY REHABILITATION AND PREVENTION, (29), 5, 314-317. http://dx.doi.org/10.1097/HCR.0b013e3181ac785f

Copyright: Lippincott, Williams \& Wilkins http://www.lww.com/

Postprint available at: Linköping University Electronic Press

http://urn.kb.se/resolve?urn=urn:nbn:se:liu:diva-51390 


\section{Brief report}

\section{Stress reduction in patients with coronary artery disease.}

Does it change the levels of cortisol secretion?

Mireille Rydén, MSc, Bo Hedbäck, MD, PhD, Lena Jonasson, MD, PhD.

Division of Cardiovascular Medicine, Department of Medical and Health Sciences, Linköping University, Linköping, Sweden.

Correspondence: Lena Jonasson, $\mathrm{MD}, \mathrm{PhD}$

Division of Cardiovascular Medicine

Department of Medical and Health Sciences

Linköping University

SE-581 85 Linköping, Sweden

Tel: +463225194

E-mail: lena.jonasson@lio.se

Total word count: 1955 (including references) 
Brief report

Stress reduction in patients with coronary artery disease.

Does it change the levels of cortisol secretion? 


\begin{abstract}
It has been shown that behavioural therapy has effects on stress behaviour in patients with coronary artery disease (CAD). Salivary cortisol measurements are widely used to assess psychological stress and/or stress reactivity. The aim of this study was to investigate whether improved stress behaviour in type A CAD patients involved changes in cortisol secretion pattern. Twenty-four male patients were identified as type A individuals and completed a 12 months cognitive-behavioral stress management program. Stress behaviour was evaluated by using a validated questionnaire. Morning and evening salivary cortisol levels were measured over 3 consecutive days at baseline and after 12 months. Although the patients showed a significant improvement in psychosocial well-being after 12 months, their basal cortisol levels or diurnal rhythm of cortisol did not change. There was no correlation between stress score and cortisol levels. The value of salivary cortisol as both a stress marker and a new cardiovascular risk factor has been discussed but the data from this small pilot study raise the question of its utility as a stress marker in cardiac rehabilitation.
\end{abstract}




\section{Introduction}

An increasing body of evidence indicates that psychosocial stress is a significant contributor to the course of cardiovascular disease. Recently, one large case-control study evaluating multiple elements of stress (the INTERHEART study) demonstrated that psychosocial stress was related to an increased risk of acute myocardial infarction (1). Prospective populationbased studies have also provided evidence for a number of emotional and environmental psychosocial risk factors, including psychological traits and states. One personality trait, the type A behaviour, has been declared an independent predictor for coronary artery disease (CAD) on the basis of several population studies (2). The type A behaviour is characterized by a number of negative emotional factors, among which hard-driving and competitive behaviour, pronounced impatience and a potential for hostility

Stress is followed by an activation of the hypothalamus-pituitary-adrenal (HPA) axis resulting in the release of corticotropin-releasing hormone by the hypothalamus. This in turn triggers the secretion of adenocorticotropic hormone (ACTH) from the pituitary gland and finally stimulates the adrenal cortex to produce cortisol. A number of studies have shown that high levels of morning cortisol are associated with high stress exposure, type A behavior and hostility (3). Other cortisol abnormalities like diurnal rhythm differences have been described in distressed individuals $(4,5)$. Interestingly, alterations in the diurnal rhythm of cortisol have also been associated with coronary artery disease and coronary calcification $(6,7)$. To determine random cortisol concentrations or the circadian pattern of cortisol secretion, measurements of free cortisol can be performed in blood or saliva. However, measurements over 2 - 6 days are considered necessary to achieve reliable trait measures, since state factors 
may bias data from a single day (8). Therefore, the determination of salivary cortisol has become the method of choice in basic research and clinical environments.

Reductions in serum or salivary cortisol or altered cortisol patterns have been demonstrated after stress management interventions (9-11). Today, many cardiac rehabilitation units offer stress management programs to patients with type A-like behaviour pattern. The aim of this pilot study was to investigate whether improved stress behaviour in type A CAD patients also involved changes in cortisol secretion.

\section{Methods}

Among patients who were admitted to the hospital-based cardiac rehabilitation unit 4-6 weeks after an index event of acute coronary syndrome, type A individuals were identified by using a short version of the Jenkins Active Survey (12). Thirty-nine consecutive patients (34 men and 5 women) agreed to participate in a stress intervention program and were all at the time of inclusion in a clinically stable condition. At baseline and after 12 months, the participants answered a questionnaire, The Everyday Life Stress Scale (13). Statements in this questionnaire are connected to either time urgency/impatience or easily aroused irritation/hostility.

The cognitive-behavioral stress management program comprised 202 -h sessions over the course of 1 year, held weekly for the first 10 weeks and then after monthly (13). Each group consisted of 7-8 patients. The program was held by nurses/psychotherapists, especially 
educated for this purpose and included five key elements; 1) education, 2) self-monitoring, 3) skills training, 4) cognitive restructuring and 5) spiritual development.

Diurnal salivary cortisol was measured at baseline and after 12 months, as previously described (7). Patients were instructed to collect two saliva samples each day on 3 consecutive days, the first sample taken 30 minutes after awakening and the second sample in the evening before going to bed. Salivette swabs (Sarstedt, Numbrecht, Germany) were placed under the tongue by the patient for 2 minutes. The Salivettes were immediately frozen at $20^{\circ} \mathrm{C}$ until analysis. Free cortisol was determined by a modified commercial radioimmunoassay (Diagnostic Products Corporation; Los Angeles, US).

Data were analyzed using SPSSPC (SPSS, Inc., Chicago. Illinois) and presented as median (interquartile range). To determine possible correlations, Pearson's correlation coefficient was used. Statistical significance was analyzed by Mann-Whitney, using the two-tailed $p$ values $<0.05$.

\section{Results}

Twenty-four male patients (median age 62 (55-66)), completed the whole program, including all assessments. At baseline, the majority of patients had been treated with beta-blockers (74\%) and statins (85\%) for at least 1 month. Two patients were on long-term treatment with selective serotonin reuptake inhibitors at inclusion. The medication did not change during the 12 months program. Clinical characteristics, like blood pressure, heart rate and waist circumference remained unchanged from baseline to 12 months. Three patients were smokers 
at baseline, one of them quitted smoking during the program. At 12 months, laboratory characteristics like low density lipoprotein cholesterol, high density lipoprotein cholesterol, triglycerides and C-reactive protein remained similar to baseline values.

The cortisol levels 30 minutes after awakening and at bedtime were similar at baseline and 12 months, as was the diurnal cortisol deviation (see Table 1).

According to the Everyday Life Stress questionnaire, patients showed a significant improvement in psychosocial well-being after 12 months (see Figure 1). However, no significant correlations between stress score and cortisol levels or between stress score and other biological markers were revealed at any time point. Neither were there any significant correlations between cortisol levels and other biological markers.

The individuals who did not complete the 12 months program, including all assessments, did not differ from the study subjects regarding stress score, cortisol 30 minutes after awakening or cortisol at bedtime.

\section{Discussion}

Stress behaviour was markedly reduced in male type A CAD patients that participated in a cognitive-behavioral stress management program. The significant improvement in self-rated stress score is in line with a recent stress intervention study evaluating the effect of cognitivebehavioral therapy in CAD. Female CAD patients reported a significantly less stress behaviour when randomized to the same 12 months cognitive-behavioral stress management 
program as we used (14). Several population-based studies have shown that type A behavior and hostility are predictors of CAD, independent of traditional risk factors (2). Moreover, there is consistent evidence that emotional stress and anger can trigger acute coronary events (15). One earlier randomized-control trial has investigated whether alteration of type A behavior in secondary prevention of CAD is associated with a decrease in cardiac recurrent rate. Post-infarction patients who were randomized to continuous type A behavior counseling showed a significantly reduced intensity of type A behavior and a similar significant decrease in both cardiac mortality and morbidity during 5 years of follow-up (16).

The basal levels of cortisol or the diurnal cortisol decline did not correlate with the self-rated stress behavior. Neither did the cortisol values show any tendency to change after the 12 months intervention program. These results were somewhat unexpected since cortisol measurements, in particular evening cortisol and the diurnal deviation of cortisol, have been found to be reliable biomarkers of stress $(4,5)$. Well-maintained reductions in single measurements of serum cortisol and a shift towards a steeper diurnal decline have also been demonstrated after stress reduction programs in men with chronic occupational stress and cancer patients (9-11). Type A behavior is related to a high level of ACTH secretion and some earlier studies have also shown that type A persons are likely to express high serum levels of cortisol $(3,17)$. However, compared to the intensity of type A behaviour considered alone, certain components of the type A behaviour like hostility and vital exhaustion have been shown to be more associated with high cortisol levels (17). This highlights the possibility that the type A patients in our study constituted a selected group without any hormonal dysfunction. In other type A patients, factors like high impatience and hostility may have caused non-compliance or even prevented them from participating in the program. However, 
neither the stress score or cortisol parameters in the 15 patients not completing the program differed from the values in the study group.

Another point worth mentioning is that a number of prospective studies in CAD populations have not shown any prognostic role for type A/hostility (2). In contrast, a significant prognostic role of other psychosocial traits like type D or "distressed" personality, anxiety, depression and low social support have been consistently reported (2). Furthermore, the type D characteristics have been associated with hypersecretion of cortisol and flattened diurnal cortisol profile $(5,18)$. Since recent studies have shown that high baseline cortisol levels and low cortisol deviation are associated with coronary atherosclerosis $(6,7)$, it has been proposed that the behavioural pattern plays a role in CAD by raising neuroendocrine responses. The value of salivary cortisol as both a stress marker and a new cardiovascular risk factor has been discussed. However, despite the obvious limitations of this pilot study related to small sample size and lack of control group, the data strongly suggest that the intensity of type A-like behaviour in CAD patients can not be reflected in salivary cortisol levels. Instead, we believe that future studies are needed to investigate whether cardiac rehabilitation focusing on other psychosocial risk behaviour has more pronounced effects on the cortisol pattern.

\section{References}

1. Rosengren A, Hawken S, Ounpuu S, et al. INTERHEART Investigators. Association of psychosocial risk factors with risk of acute myocardial infarction in 11119 cases and 13648 controls from 52 countries (the INTERHEART study): case-control study. Lancet 2004; 364:953-62. 
2. Hemingway H, Marmot M. Psychosocial factors in the aetiology and prognosis of coronary heart disease: systematic review of prospective cohort studies. BMJ 1999; 318:1460-7.

3. Steptoe A Cropley M, Griffith J, Kirschbaum C. Job strain and anger expression predict early morning elevations in salivary cortisol. Psychosom Med 2000;62:286-92.

4. Cohen S, Schwartz JE, Epel E, Kirschbaum C, Sidney S, Seeman T. Socioeconomic status, race and diurnal cortisol decline in the Coronary Artery Risk Development in Young Adults (CARDIA) Study. Psychosom Med 2006;68:41-50.

5. Sjögren E, Leanderson P, Kristenson M. Diurnal saliva cortisol levels and relations to psychosocial factors in a population sample of middle-aged Swedish men and women. Int J Behav Med 2006;13,193-200.

6. Matthews K, Schwartz J, Cohen S, Seeman T. Diurnal cortisol decline is related to coronary calcification: CARDIA study. Psychosom Med 2006;68:657-61.

7. Nijm J, Kristenson M, Olsson AG, Jonasson L. Impaired cortisol response to acute stressors in patients with coronary disease. Implications for inflammatory activity. $\mathbf{J}$ Intern Med, 2007; 262:375-84.

8. Kirschbaum C, Hellhammer DH. Salivary cortisol in psychoneuroendocrine research: Recent developments and applications. Psychoneuroendocrinology 1994;19:313-33.

9. Nickel C, Tanca S, Kolowos S, et al. Men with chronic occupational stress benefit from behavioural/psycho-educational group training: a randomized, prospective, controlled trial. Psychol Med 2007;37:1141-9.

10. Phillips KM, Antoni MH, Lechner SC, et al. Stress management intervention reduces serum cortisol and increases relaxation during treatment for nonmetastatic breast cancer. Psychosom Med 2008; Epub ahead of print. 
11. Carlson L, Speca M, Patel K, Goodey E. Mindfulness-based stress reduction in relation to quality of life, mood, symptoms of stress and levels of cortisol, dehydroepiandrosterone sulphate (DHEAS) and melatonin in breast and prostate cancer outpatients. Psychoneuroendocrinology 2004;29:448-74.

12. Jenkins CD, Rosenman RH, Friedman M. Development of an objective psychological test for the determination of coronary-prone behaviour pattern in employed men. J Chron Dis 1967;20:371-9.

13. Burell G, Granlund B. Women’s hearts need special treatment. Int J Behav med 2002;9:228-42.

14. Claesson M, Birgander LS, Lindahl B, et al. Women's hearts - stress management for women with ischemic heart disease: explanatory analyses of a randomized controlled trial. J Cardiopulm Rehabil 2005;25:93-102.

15. Strike PC, Steptoe A. Behavioral and emotional triggers of acute coronary syndromes: a systematic review and critique. Psychosom Med 2005;67:179-86.

16. Friedman M, Thoresen CE, Gill JJ, et al. Alteration of type A behaviour and its effects on cardiac recurrences in post myocardial infarction patients: summary results of the recurrent coronary prevention project. Am Heart J 1986;112:653-65.

17. Keltikangas-Järvinen L, Räikkönen K, Hautanen A. Type A behavior and vital exhaustion as related to the metabolic hormonal variables of the hypothalamic-pituitary-adrenal axis. Behav Med 1996;22:15-22.

18. Whitehead DL, Perkins-Porras L, Strike PC, Magid K, Steptoe A. Cortisol awakening response is elevated in acute coronary syndrome patients with type-D personality. $\mathrm{J}$ Psychosom Res 2007; 62:419-25. 
Table 1. Salivary cortisol levels (nmol/l) and diurnal cortisol deviation at baseline and after 12 months.

\begin{tabular}{llll}
\hline & Baseline & 12 months & P-value \\
& $(\mathrm{n}=24)$ & $(\mathrm{n}=24)$ & \\
& & & \\
\hline 30 min after awakening & $11.7(9.3-14.2)$ & $10.9(8.2-15.3)$ & $\mathrm{NS}$ \\
& & & \\
Evening & $1.4(0.8-2.0)$ & $1.6(1.0-2.7)$ & $\mathrm{NS}$ \\
& & & \\
Diurnal deviation & $10.1(8.2-12.5)$ & $9.8(6.3-14.4)$ & $\mathrm{NS}$ \\
& & & \\
\hline
\end{tabular}

Values are given as median (interquartile range). The cortisol values are given as the unit $\mathrm{nmol} / \mathrm{l}$. Diurnal deviation $=$ mean value of morning cortisol level minus mean value of evening cortisol level. 
Figure 1. The result from The Everyday Life Stress Questionnaire at baseline and after 12 months. The stress score ranges between 0 and 60 points and higher score indicates higher stress behavior.

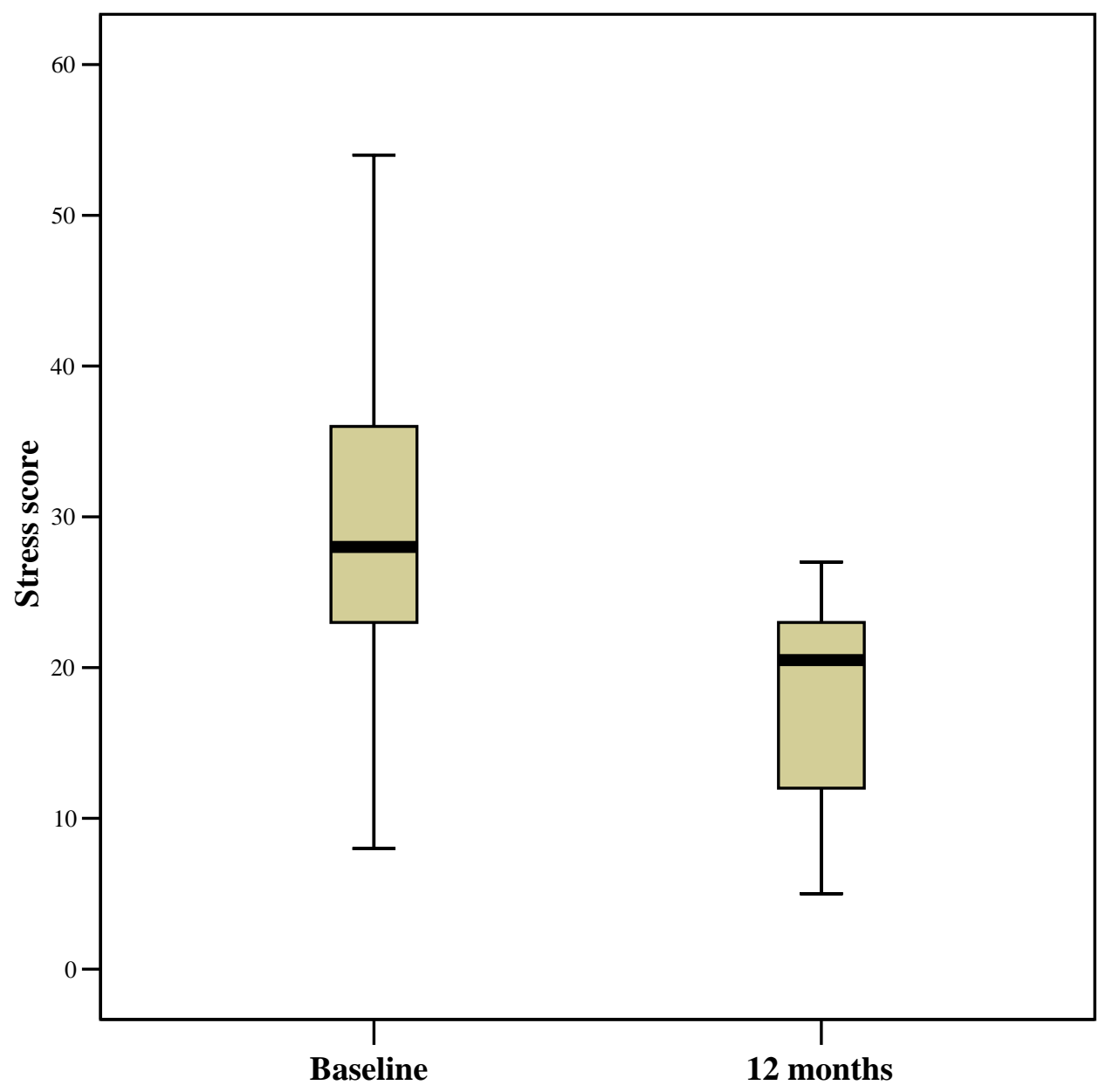


